\title{
Comparação das distâncias previstas e percorridas no incremental shuttle walking test em idosos comunitários
}

\author{
Comparison of predicted and covered distances in the incremental shuttle walking test in \\ older adults
}
Comparación de las distancias predichas y recorridas en la incremental shuttle walking test en ancianos comunitarios
Adriana Netto Parentoni', Mayana Marques Simões², Valéria Cristina de Faria³, Giane Amorim Ribeiro Samora ${ }^{4}$ Danielle Aparecida Gomes Pereira ${ }^{5}$, Lygia Paccini Lustosa (in memoriam) ${ }^{6}$

RESUMO I O objetivo deste estudo foi avaliar a validade externa de três equações de referência para o cálculo da distância a ser percorrida no incremental shuttle walking test (ISWT) por idosos comunitários, e, por fim, identificar qual equação prediz melhor a distância nessa faixa etária. Foram selecionados 84 idosos cadastrados nas Unidades Básicas de Saúde de Diamantina, Minas Gerais. Os voluntários foram submetidos ao ISWT e a distância percorrida em metros até a interrupção do teste foi registrada e utilizada para comparação com as equações de predição. Os voluntários apresentaram média de idade de 73,15 $\pm 5,89$ anos e alguns comprometimentos de saúde comuns dessa faixa etária, sendo a maioria mulheres e praticantes de atividade física. A análise estatística demonstrou que houve interação entre os fatores, ou seja, existe diferença entre as distâncias obtidas pelo ISWT e as distâncias obtidas pelas equações. Houve diferença também nas comparações entre os sexos $(p<0,05)$ e a Equação 1 apresentou maior força de correlação $(p<0,0001 ; r=0,414)$ com a distância real percorrida em relação às outras equações. Portanto, este estudo demonstrou uma diferença na comparação das distâncias real e calculadas por três equações de predição do ISWT, sugerindo que as equações utilizadas podem superestimar a distância percorrida pelos idosos, e que entre essas a Equação 1 apresentou uma predição mais próxima da distância percorrida para a amostra de idosos avaliada. Descritores / Idoso; Predição; Teste de Caminhada; ISWT.

\begin{abstract}
I Our study aimed to evaluate the external validity of three reference equations for estimating the distance to be covered in the incremental shuttle walking test (ISWT) by older adults and to identify which equation best predicts the distance for this age group. In total, 84 older adults registered in the Health Centers of Diamantina, state of Minas Gerais, Brazil, were selected. The volunteers were subjected to the ISWT and the distance covered, in meters, until the interruption of the test was recorded and compared with the prediction equations. The volunteers had a mean age of $73.15 \pm 5.89$ years and some health problems common to this age group, were mostly women, and physically active. Statistical analysis showed an interaction between the factors, that is, a difference between the distances covered the ISWT and the distances obtained by the equations. The analysis showed a difference between the men and women $(p<0.05)$ and Equation 1 showed a greater correlation
\end{abstract}

\footnotetext{
'Universidade Federal dos Vales do Jequitinhonha e Mucuri (UFVJM) - Diamantina (MG), Brasil. E-mail: adrianaparentoni@gmail.com. Orcid: 0000-0002-2105-4265

2Universidade Federal dos Vales do Jequitinhonha e Mucuri (UFVJM) - Diamantina (MG), Brasil. E-mail: mayana.simoes9@gmail.com. Orcid: 0000-0001-9382-6626

¿Universidade Federal de Minas Gerais (UFMG) - Belo Horizonte (MG), Brasil. E-mail: valeriaefiufv@yahoo.com.br. Orcid: 0000-0003-4816-439X

${ }^{4}$ Universidade Federal de Minas Gerais (UFMG) - Belo Horizonte (MG), Brasil. E-mail: gribeirosamora@gmail.com. Orcid: 0000-0001-5102-369X

${ }_{5}^{5}$ niversidade Federal de Minas Gerais (UFMG) - Belo Horizonte (MG), Brasil. E-mail: danielleufmg@gmail.com. Orcid: 0000-0003-4400-2326

${ }^{6}$ Universidade Federal de Minas Gerais (UFMG) - Belo Horizonte (MG), Brasil. E-mail: lygia.paccini@gmail.com.

Orcid: 0000-0002-0919-1320
} 
strength ( $p<0.0001 ; r=0.414)$ with the distance covered compared to the other equations. Therefore, our study showed a difference in the comparison of the distances covered and those estimated by three ISWT prediction equations, suggesting that the equations used may overestimate the distance covered by older adults, and that, Equation 1 showed a prediction closer to the distance covered by the older adults of the sample.

Keywords | Aged; Forecast; Walk Test; ISWT.

RESUMEN | El objetivo de este estudio fue evaluar la validez externa de tres ecuaciones de referencia para calcular la distancia recorrida de la incremental shuttle walking test (ISWT) para ancianos comunitarios, así como identificar la ecuación que mejor predice la distancia en este grupo de edad. Se seleccionaron a 84 ancianos inscritos en las Unidades Básicas de Salud de Diamantina, Minas Gerais (Brasil). Se aplicaron a los voluntarios la ISWT, y la distancia recorrida en metros hasta la interrupción de la prueba se registró y se utilizó para comparar con las ecuaciones de predicción. Los voluntarios tenían un promedio de edad de 73,15ะ5,89 años y algunos problemas de salud habituales en este grupo de edad, de los cuales la mayoría fue mujeres y practicantes de actividad física. El análisis estadístico mostró que hubo una interacción entre los factores, es decir, existe una diferencia entre las distancias obtenidas por ISWT y las distancias obtenidas por las ecuaciones. También hubo una diferencia en las comparaciones entre los sexos $(p<0,05)$, y la Ecuación 1 mostró una mayor fuerza de correlación ( $p<0,0001$; $r=0,414)$ con la distancia real recorrida en relación con las otras ecuaciones. Por lo tanto, este estudio demostró una diferencia en la comparación de las distancias reales y calculadas por tres ecuaciones de predicción de ISWT, lo que sugiere que las ecuaciones utilizadas pueden sobreestimar la distancia recorrida por los ancianos y que entre estas la Ecuación 1 presentó una predicción más cercana a la distancia recorrida por la muestra evaluada.

Palabras clave | Anciano; Predicción; Prueba de Caminata; ISWT.

\section{INTRODUÇÃO}

A população idosa mundial apresenta um ritmo de crescimento acelerado. Em 2008, o Brasil atingiu 20 milhões de idosos e as projeções apontavam para um aumento crescente. A expectativa é que em 2025 o Brasil ocupe a sexta posição em países com maior número de idosos, contabilizando $15 \%$ da sua população ${ }^{1,2}$. Esse envelhecimento acelerado implica grandes desafios para o cuidado e tem despertado a necessidade de estudos e pesquisas na área ${ }^{3,4}$, incluindo estudos clínicos e metodológicos, para unificação e padronização de medidas e avaliações.

O processo de envelhecimento é um fenômeno fisiológico e de alta complexidade durante o qual ocorrem prejuízos graduais na capacidade dos indivíduos de manter suas funções básicas ${ }^{5}$. Nesse sentido, a capacidade funcional é entendida como a condição de aptidão para manter-se capaz de exercer as atividades básicas e instrumentais de vida diária, suficientes e inerentes a uma vida com independência ${ }^{6}$. Dessa forma, a avaliação do desempenho funcional torna-se uma medida imprescindível para esse público. Assim, medidas de capacidade funcional são utilizadas na prática clínica e científica para inferir no estado de saúde global, acompanhamento do estado clínico, avaliação de incapacidade física e determinação dos níveis de dependência ${ }^{7}$.
$\mathrm{Na}$ literatura existem vários instrumentos de medida para avaliação do desempenho funcional do idoso, tais como questionários e testes de desempenho ${ }^{7,8}$, sendo sua escolha baseada no objetivo e ambiente disponível para sua aplicação. Dentre os testes de desempenho destaca-se o incremental shuttle walking test (ISWT) ${ }^{9}$, uma adaptação do shuttle run test ${ }^{10}$. O ISWT é um teste simples, progressivo, de baixo custo, que avalia a capacidade funcional aeróbia dos indivíduos e é aplicável a diversas faixas etárias ${ }^{11}$. Assim, a partir da sua ampla aplicação e com o intuito de prever a distância ideal a ser percorrida pelo indivíduo no ISWT, alguns autores propuseram equações de referência para a população brasileira ${ }^{12-15}$. Nesse contexto, apenas três baseiam-se no mesmo tipo de variáveis, antropométricas e demográficas, que foram estabelecidas após utilização de um mesmo protocolo de execução ${ }^{12-14}$. No entanto, existe o pressuposto de que ao utilizar equações para previsão da distância a ser percorrida no ISWT pode-se subestimar ou superestimar essa distância. Essa interpretação pode gerar erros e condutas inadequadas pelos profissionais e pesquisadores ${ }^{13}$ e deve ser mais bem investigada, principalmente para a população de idosos.

A importância do ISWT reside no fato de ele ser usado para avaliar o resultado de intervenções ${ }^{9,11}$, quantificar a capacidade funcional ${ }^{11,16,17}$ e determinar prognósticos ${ }^{11,16,18}$. Mas, embora o ISWT seja utilizado em diversas condições de saúde e populações, inclusive em idosos, não há na literatura artigos que apontem qual 
das equações existentes estima de forma mais aproximada a distância a ser percorrida.

O estudo de revisão da literatura de Monteiro et al. ${ }^{11}$ demonstrou a existência de três equações para cálculo da distância percorrida no ISWT. Sendo assim, diante da importância de avaliar adequadamente a capacidade funcional no público idoso, da facilidade de realização do ISWT e da inexistência de estudos que comparem dentro da mesma população os resultados obtidos através dessas três equações distintas, que incluam homens e mulheres idosas, o objetivo deste estudo foi avaliar a validade externa das três equações de referência, e, por fim, identificar qual equação prediz melhor a distância percorrida no ISWT nessa faixa etária.

\section{METODOLOGIA}

Este estudo transversal foi realizado no município de Diamantina, Minas Gerais, com idosos cadastrados nas cinco principais Unidades Básicas de Saúde (UBS) da cidade. Faz parte de um estudo multicêntrico aprovado pelos Comitês de Ética em Pesquisa da Universidade Federal dos Vales do Jequitinhonha e Mucuri (UFVJM) e da Universidade Federal de Minas Gerais (UFMG) sob os pareceres de número 375.689 e 349.141, respectivamente, CAAE: 14129513.7 .1001 .5149 , e que aconteceu no período de 2013 a 2016.

\section{Seleção da amostra}

A Figura 1 apresenta o fluxograma de seleção da amostra. Os idosos cadastrados no ano de 2015 nas UBS receberam uma visita domiciliar e responderam a um questionário com informações clínicas, sociodemográficas e sobre a prática de atividade física, e ao teste mini exame do estado mental ${ }^{19}$. Foram considerados praticantes de atividade física aqueles que autorrelaram a prática regular de qualquer modalidade pelo menos duas vezes por semana. Foram incluídos idosos com 65 ou mais anos de idade, de ambos os sexos, que não relataram internações hospitalares nos últimos seis meses e sintomas de claudicação intermitente, e que não apresentaram déficits físicos e cognitivos que impedissem a realização do teste físico proposto. Os indivíduos elegíveis assinaram o Termo de Consentimento Livre e Esclarecido e foi agendado o dia de realização do ISWT. Foram excluídos da amostra os indivíduos que não compareceram para a realização do teste e os que não o realizaram.
1096 idosos cadastrados e visitados em domicílio

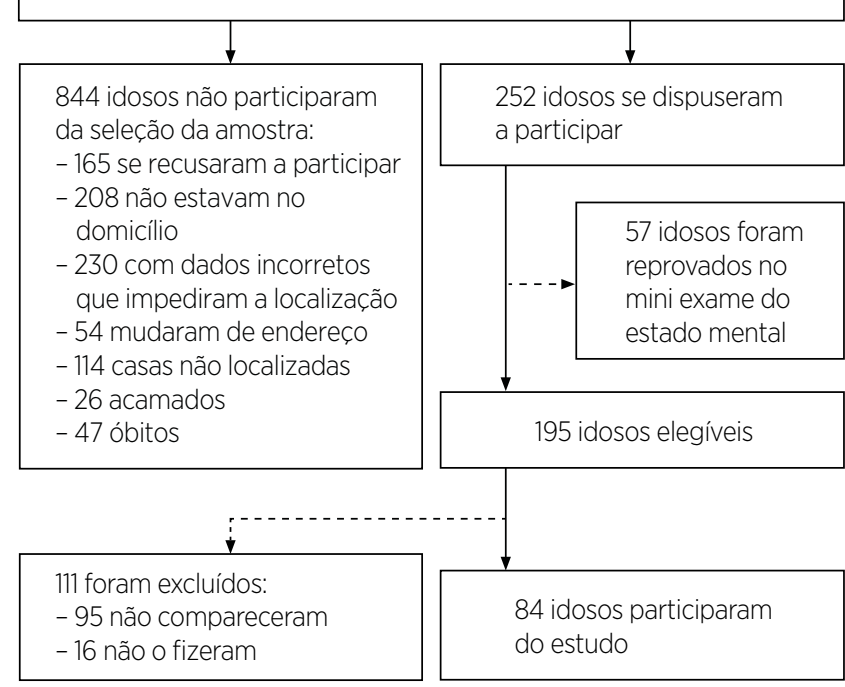

Figura 1. Fluxograma de seleção da amostra

\section{Procedimentos}

Os voluntários foram submetidos ao ISWT, um teste de velocidade de caminhada progressiva e com mudança de direção a partir da orientação de diferentes sinais sonoros ${ }^{9}$. O ISWT foi realizado em locais próximos às residências dos idosos, em um corredor de 10 metros, plano, com piso sem irregularidades. Nove metros foram delimitados por dois cones e meio metro após cada cone, para que o indivíduo realizasse o contorno ${ }^{11}$. O teste foi interrompido quando o indivíduo não alcançou dois cones de forma consecutiva $^{15,20}$, quando apresentou sintomas limitantes e quando atingiu valores de $85 \%$ da frequência cardíaca máxima $\left(\mathrm{FC}_{\text {Máx }}\right)$, prevista pela fórmula (220-idade $)^{9,20}$. Pelo fato de o ISWT ter sido realizado em locais próximos às residências dos idosos e não dentro de instituições com a presença de equipe médica, optou-se, por questões de segurança, pelo protocolo submáximo de 12 estágios. Essa escolha evitou a fadiga extrema dos participantes e garantiu que não houvesse nenhuma intercorrência durante a realização dos testes. A distância percorrida, em metros, até a interrupção do teste foi registrada e utilizada para comparação com as equações de predição.

De acordo com a literatura, a capacidade funcional de idosos é diferente entre os sexos, sendo menor nas mulheres ${ }^{21,22}$. Dessa forma, as distâncias foram comparadas considerando a amostra total e divididas por sexo. Além disso, ter 70 anos ou mais está associado à incapacidade funcional ${ }^{22}$. Portanto, para avaliar o impacto da idade na capacidade de predição das equações também foi avaliada a relação entre idade e a diferença entre a distância real e as distâncias previstas. 
Assim, para a predição da distância percorrida no ISWT foram utilizadas três equações descritas na literatura e utilizadas para a população brasileira ${ }^{12-14}$. Essa escolha baseou-se no fato de elas utilizarem o mesmo protocolo de aplicação do teste deste estudo:

(1) ISWTprev=374,004-(6,782*idade (anos) $)-\left(2,328^{*}\right.$ peso $(\mathrm{kg}))+\left(3,865^{*}\right.$ altura $\left.(\mathrm{cm})\right)+\left(115,937^{*} \text { sexo }^{\#}\right)^{12}$;

(2) ISWTprev $=34,608-(4,384 *$ idade(anos $))-\left(2,949^{*}\right.$ peso $(\mathrm{kg}))+\left(553,336^{*}\right.$ altura $\left.(\mathrm{m})\right)+\left(114,387^{*} \text { sexo }^{\#}\right)^{13}$;

(3) ISWTprev=347,7-(7,2*idade(anos) $)-(3 *$ peso $(\mathrm{kg}))+$ $\left(472,3^{*}\right.$ altura $\left.(\mathrm{m})\right)+\left(137,2^{*} \operatorname{sexo}^{\#}\right)^{14}$.

\section{Análise estatística}

A distribuição normal dos dados foi verificada por meio do teste de Shapiro-Wilk, que detectou distribuição simétrica. As comparações entre a distância percorrida no ISWT e as distâncias previstas a partir das três equações, entre os sexos masculino e feminino, e a interação entre esses fatores, foram analisadas por meio da análise de variância (Anova) fatorial mista. O teste post hoc adotado foi o de Bonferroni para múltiplas comparações. A relação entre os valores previstos pelas equações e o valor real obtido pelo ISWT, assim como a relação entre a idade e a diferença entre os valores obtidos e previstos por cada equação, foram avaliados pelo teste de correlação de Pearson. O nível de significância alfa adotado foi de 5\% e, para análise dos dados, foi utilizado o programa Statistical Package for the Social Science (SPSS 19.0).

Para o cálculo amostral foi considerado um estudo piloto realizado com 10 idosos, que demonstrou um tamanho de efeito moderado $\left(\eta^{2}=0,29\right)$, estabelecido um poder de $80 \%$ e um nível de significância alfa de $5 \%$, demonstrando a necessidade de um total de 18 indivíduos para compor a amostra.

\section{RESULTADOS}

Participaram deste estudo 84 indivíduos com média de idade de $73,15 \pm 5,89$ anos. A maioria dos participantes eram mulheres e praticantes de atividade física. As características da amostra estão descritas na Tabela 1. As condições de saúde presentes na amostra foram: déficit visual $(86,90 \%)$, hipertensão arterial $(77,38 \%)$, déficit auditivo (50\%), osteoartrite (25\%), deformidade nos pés $(21,43 \%)$ e perda de memória (19,05\%).

\begin{tabular}{|c|c|}
\hline Características & n (\%) \\
\hline \multicolumn{2}{|l|}{ Sexo } \\
\hline Feminino & $66(78,57)$ \\
\hline Masculino & $18(21,43)$ \\
\hline \multicolumn{2}{|l|}{ Idade (anos) } \\
\hline 65 a 69 & $23(27,38)$ \\
\hline 70 a 79 & $48(57,14)$ \\
\hline 80 a 89 & $13(15,48)$ \\
\hline \multicolumn{2}{|l|}{ Atividade Física } \\
\hline Sim & $50(59,52)$ \\
\hline Não & $34(40,48)$ \\
\hline
\end{tabular}

A Tabela 2 apresenta as distâncias obtidas a partir da realização do ISWT e das três equações de referência adotadas. A análise estatística demonstrou que houve interação entre os fatores, ou seja, existe diferença entre as distâncias obtidas pelo ISWT e as distâncias obtidas pelas equações. Houve diferença também nas comparações entre os sexos $(\mathrm{p}<0,05)$. Considerando a amostra total, a distância real obtida pelo ISWT atingiu 58,82\%, 50,80\% e 53,14\% das distâncias previstas pelas Equações 1,2 e 3, respectivamente. Além disso, destaca-se que não houve correlação significativa entre a idade e a diferença entre as distâncias percorrida pelo ISWT e pela distância prevista por cada uma das equações (Equação 1: $p=0,896$; equação 2: $\mathrm{p}=0,223$; equação 3: $\mathrm{p}=0,986)$.

Tabela 2. Distâncias percorridas no ISWT e previstas pelas três equações, considerando amostra total e amostra dividida por sexo

$\begin{array}{lccc} & \text { Total }(\mathrm{n}=84) & \begin{array}{c}\text { Feminino } \\ (\mathrm{n}=66)\end{array} & \begin{array}{c}\text { Masculino } \\ (\mathrm{n}=18)\end{array} \\ \begin{array}{l}\text { Distância real } \\ \text { no ISWT }(\mathrm{m})\end{array} & 208,47 \pm 111,78 & 195,69 \pm 103,20 & 255,33 \pm 131,60^{\#} \\ \text { Equação } 1^{12}(\mathrm{~m}) & 354,40 \pm 83,26^{\circ} & 320,97 \pm 52,62^{8} & 477,00 \pm 54,79^{\beta} \\ \text { Equação } 2^{13}(\mathrm{~m}) & 409,90 \pm 91,33^{\circ} & 371,31 \pm 53,20^{\&} & 551,40 \pm 54,02^{\beta} \\ \text { Equação } 3^{14}(\mathrm{~m}) & 392,31 \pm 97,83^{\circ} & 352,29 \pm 60,04^{8} & 539,07 \pm 62,60^{\beta}\end{array}$

ISWT: Incremental Shuttle Walking Test. Dados representados por valores de média (desvio-padrão). 'Diferença significativa em relação à distância real no ISWT na amostra total ( $p<0,001$ ); "Diferença significativa entre os sexos para a distância real no ISWT $(p<0,001)$; ${ }^{8}$ Diferença significativa em relação à distância real no ISWT no sexo feminino ( $p<0,001)$; RDiferença significativa em relação à distância real no ISWT no sexo masculino ( $p<0,001)$.

A Figura 2 mostra que houve correlação significativa entre as distâncias previstas pelas três equações e a distância real obtida pelo ISWT ( $\mathrm{p}<0,0001)$. No entanto, a Equação 1 apresentou correlação mais forte $(r=0,414)$ em relação às outras. 

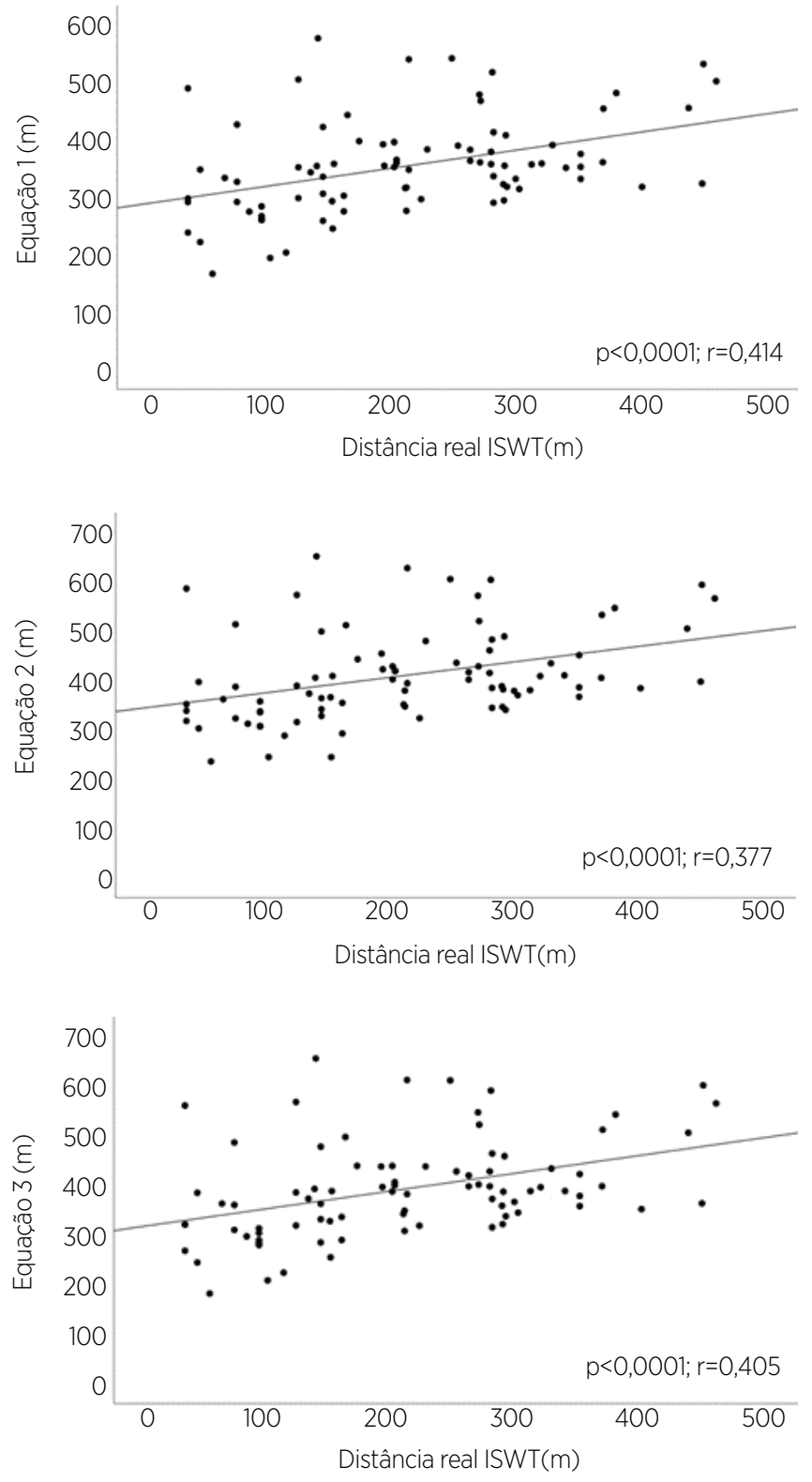

Figura 2. Correlação entre a distância real obtida pelo ISWT e as distâncias previstas por cada equação

Em relação à realização do ISWT, nenhum dos idosos concluiu os 12 estágios previstos no teste e as causas de interrupção foram: 61 idosos $(72,62 \%)$ não atingiram dois cones consecutivos, 21 idosos (25\%) atingiram $85 \%$ da $\mathrm{FC}_{\text {Máx }}$ e dois idosos $(2,38 \%)$ apresentaram sintomas limitantes de dor.

\section{DISCUSSÃO}

Este estudo evidenciou que há diferença significativa entre as distâncias previstas pelas três equações avaliadas e a distância real percorrida no ISWT. Da mesma forma, houve diferença na análise quando a amostra foi dividida por sexo. Essas diferenças apontaram que a distância percorrida foi estatisticamente menor que a prevista pelas três equações, sugerindo que a utilização dessas equações de referência, para o público idoso, pode superestimar a distância real a ser percorrida.

Inicialmente, é importante ressaltar que os estudos que propuseram as equações ${ }^{12-14}$ não contaram com uma amostra exclusivamente idosa, avaliando indivíduos acima de 40 anos, e apenas saudáveis; e que para o protocolo do ISWT adotaram 15 estágios com a intenção de evitar o efeito teto, e o consideraram como um teste máximo. Portanto, não foi adotado como critério de interrupção do teste atingir $85 \%$ da $\mathrm{FC}_{\text {Máx }}$.

Considerando o ISWT como um teste máximo, Soares et al. ${ }^{23}$ estudaram mulheres, obesas de 18 a 65 anos (média de 44 anos) e identificaram diferença significativa de 146 metros entre a distância percorrida e a prevista pela equação de Jurgensen et al. ${ }^{12}$.Já este estudo analisou exclusivamente indivíduos com 65 anos ou mais que apresentavam condições de saúde comuns para sua faixa etária ${ }^{24}$, e cujo protocolo escolhido foi de caráter submáximo, como já relatado anteriormente. Sendo assim, a diferença nas características das amostras de ambos os estudos, obesos ou outras condições de saúde, pode justificar a diferença entre as distâncias previstas pelas equações e obtida no ISWT.

Além disso, este estudo possui mais de $70 \%$ da sua amostra com mais de 70 anos, e, apesar de a literatura apontar que a incapacidade funcional está associada a essa faixa etária ${ }^{22}$, não foi identificada correlação significativa entre idade e diferença das distâncias previstas e obtidas no ISWT, o que reforça que a condição de saúde da amostra pode ser a principal explicação para a diferença entre as distâncias previstas e obtidas.

Outro ponto importante a se considerar na amostra deste estudo é a uma maior proporção de idosos praticantes de atividade física, o que implicaria melhor desempenho no ISWT. Entretanto, isso não foi apoiado pelos resultados, o que possivelmente se deve à superestimação da prática de atividade física avaliada por autorrelato. Dessa forma, comprometimentos de saúde importantes e comuns nessa faixa etária, como os apresentados pela amostra deste estudo, devem ser considerados ao se propor uma equação para idosos que se pretenda uma maior validade externa.

Em relação à diferença entre sexos, os dados demonstrados aqui estão em concordância com os resultados de Jüngensen et al. ${ }^{12}$ e Dourado, Vidotto e Guerra ${ }^{13}$, que observaram que homens percorreram distâncias significativamente maiores no ISWT em relação às mulheres. Essa diferença em relação à distância 
pode ser explicada pelo fato de que os homens apresentam maior força, massa muscular e maior estatura ${ }^{12}$. Nesse caso, a relação de força muscular e tamanho da passada deve ser investigada em estudos futuros.

O maior número de indivíduos do sexo feminino neste estudo reflete o fenômeno da "feminização na velhice", que ocorre devido ao maior índice de mortalidade masculina ${ }^{25}$. Além disso, está de acordo com outros estudos ${ }^{21,26,27}$ e com o Censo $2010^{28}$ quanto à maior proporção de mulheres em relação aos homens no Brasil. Essa diferença no número amostral entre homens e mulheres não afetou os resultados, uma vez que as três equações analisadas consideram a variável sexo para o cálculo.

Este estudo apresenta algumas limitações. Uma delas refere-se à escolha do protocolo original do ISWT com 12 níveis e submáximo. Apesar de os estudos que originaram as equações de predição terem utilizado o protocolo adaptado de 15 níveis e máximo, essa escolha pareceu ser a mais segura e adequada para o tipo de população estudada. Importante ainda ressaltar que nenhum idoso completou todos os estágios, o que reforça que o protocolo adotado foi adequado e seguro para essa população. Outra limitação foi o ISWT ter sido realizado apenas uma vez neste estudo, diferentemente dos estudos que propõem as equações. No entanto, essa conduta foi tomada para se adequar ao contexto de pesquisa na comunidade, na qual comumente a disponibilidade de tempo e recursos são restritas. Em contrapartida, um ponto positivo deste estudo refere-se ao fato de que o ISWT foi aplicado pelo mesmo avaliador e com seu protocolo original, o que reforça a confiabilidade da sua aplicação.

\section{CONCLUSÃO}

Este estudo demonstrou diferença na comparação das distâncias real e calculada por três equações de predição do ISWT, sugerindo que as equações utilizadas podem superestimar a distância percorrida por idosos com alguns comprometimentos de saúde. No entanto, entre essas, a Equação 1 apresentou uma predição mais próxima da distância percorrida para a amostra de idosos avaliada. Esses resultados apontam para a necessidade de novos estudos específicos para essa população.

\section{AGRADECIMENTOS}

Agradecemos ao apoio do Conselho Nacional de Desenvolvimento Científico e Tecnológico (CNPq);
Fundação de Amparo à Pesquisa do Estado de Minas Gerais (Fapemig) e Coordenação de Aperfeiçoamento de Pessoal de Nível Superior (Capes).

\section{REFERÊNCIAS}

1. Veras R. Envelhecimento populacional contemporâneo: demandas, desafios e inovações. Rev Saúde Pub. 2009;43(3):548-54. doi:10.1590/S0034-89102009005000025

2. Instituto Brasileiro de Geografia e Estatística. Sinopse do Censo Demográfico de 2010 [Internet]. Rio de Janeiro: IBGE; 2011 [cited 2021 May 19]. Available from: https://biblioteca.ibge. gov.br/visualizacao/livros/liv49230.pdf

3. Miranda GMD, Mendes ACG, Silva ALA. O envelhecimento populacional brasileiro: desafios e consequências sociais atuais e futuras. Rev Bras de Geriatr Gerontol. 2016;19(3):507-19. doi:10.1590/1809-98232016019.150140

4. Brito TA, Fernandes MH, Coqueiro RS, Jesus CL, Freitas R. Capacidade funcional e fatores associados em idosos longevos residentes em comunidade: estudo populacional no Nordeste do Brasil. Fisioter Pesq. 2014;21(4):308-13. doi: 10.1590/ S0103-21002012000600017

5. Silva JP Jr, Silva LJ, Andrade DR, Oliveira LC, Santos M, Matsudo VKR. Estabilidade das variáveis de aptidão física e capacidade funcional de mulheres fisicamente ativas de 50 a 89 anos. Rev Bras Cineantropom Desempenho Hum. 2011;13(1):8-14. doi: 10.5007/1980-0037.2011v13n1p8

6. Ferreira OGL, Maciel SC, Costa SMG, Silva AO, Moreira MSP. Envelhecimento ativo e sua relação com a independência funcional. Texto Contexto Enferm. 2012;21(3):513-8. doi: 10.1590/ S0104-07072012000300004

7. Paixão CM Jr, Reichenheim ME. Uma revisão sobre instrumentos de avaliação do estado funcional do idoso. Cad Saúde Pública. 2005;21(1):7-19. doi: 10.1590/S0102-311X2005000100002

8. Camara FM, Gerez AG, Miranda MLJ, Velardi M. Capacidade funcional do idoso: formas de avaliação e tendências. Acta Fisiatr. 2008;15(4):249-56.

9. Singh SJ, Morgan MD, Scott S, Walters D, Hardman AE Development of a shuttle walking test of disability in patients with chronic airways obstruction. Thorax. 1992;47(12):1019-24. doi: 10.1136/thx.47.12.1019

10. Leger LA, Lambert J. A maximal multistage 20-m shuttle run test to predict VO2 max. Eur J Appl Physiol Occup Physiol. 1982;49(1):1-12. doi: 10.1007/BF00428958

11. Monteiro DP, Britto RR, Carvalho MLV, Montemezzo D, Parreira VF, Pereira DAG. Shuttle walking test como instrumento de avaliação da capacidade funcional: uma revisão da literatura. Rev Ciência \& Saúde. 2014;7(2):92-7. doi: 10.15448/1983-652X.2014.2.16580

12. Jürgensen SP, Antunes LC, Tanni SE, Banov MC, Lucheta PA, Bucceroni AF, et al. The incremental shuttle walk test in older Brazilian adults. Respiration. 2011;81(3):223-8. doi: 10.1159/000319037

13 Dourado VZ, Vidotto MC, Guerra RLF. Reference equations for the performance of healthy adults on field walking tests. J Bras Pneumol. 2011;37(5):607-14. doi: 10.1590/ S1806-37132011000500007 
14. Dourado VZ, Guerra RLF, Tanni SE, Antunes LCO, Godoy I. Reference values for the incremental shuttle walk test in healthy subjects: from the walk distance to physiological responses. J Bras Pneumol. 2013;39(2):190-7. doi: 10.1590/ S1806-37132013000200010

15. Probst VS, Hernandes NA, Teixeira DC, Felcar JM, Mesquita RB, Gonçalves CG, et al. Reference values for the incremental shuttle walking test. Respir Med. 2011;106(2):243-8. doi: 10.1016/ j.rmed.2011.07.023

16. Pulz C, Diniz RV, Alves AN, Tebexreni AS, Carvalho AC, Paola $A A$, et al. Incremental shuttle and six-minute walking test in the assessment of functional capacity in chronic heart failure. Can J Cardio. 2008;24(2):131-5. doi: 10.1016/s0828-282x(08)70569-5

17. Pepin V, Brodeur J, Lacasse Y, Milot J, Leblanc P, Whittom F, et al. Six-minute walking versus shuttle walking: responsiveness to broncodilatation in chronic obstructive pulmonary disease. Thorax. 2007;62(4):291-8. doi: 10.1136/thx.2006.065540

18. Morales FJ, Montemayor T, Martinez A. Shuttle versus sixminute walk test in the prediction of outcome in chronic heart failure. Int J Cardiol. 2000;76(2-3):101-5. doi: 10.1016/ s0167-5273(00)00393-4

19. Folstein MF, Folstein SE, McHugh PR. "Mini-mental state": a practical method for grading the cognitive state of patients for the clinician. J Psychiatr Res. 1975;12(3):189-98. doi: 10.1016/0022-3956(75)90026-6

20. Parreira VF, Janaudis-Ferreira T, Evans RA, Mathur S, Goldstein RS, Brooks D. Measurement properties of the incremental shuttle walk test: a systematic review. Chest. 2014;145(6): 1357-69. doi: 10.1378/chest.13-2071

21. Campos ACV, Almeida MHM, Campos GV, Bogutchi TF. Prevalência de incapacidade funcional por gênero em idosos brasileiros: uma revisão sistemática com metanálise. Rev Bras Geriatr Gerontol. 2016;19(3):545-59. doi: 10.1590/1809-98232016019.150086

22. Fiedler MM, Peres KG. Capacidade funcional e fatores associados em idosos do Sul do Brasil: um estudo de base populacional. Cad Saúde Pública. 2008;24(2):409-15. doi: 10.1590/S0102-311X2008000200020

23. Soares KKD, Gomes ELFD, Beani A Jr, Oliveira LVF, Sampaio LMM, Costa D. Avaliação do desempenho físico e funcional respiratório em obesos. Fisioter Mov. 2011;24(4):697-704. doi: 10.1590/S0103-51502011000400014

24. Gonzalez ACT, Ignácio ZM, Jornada LC, Reus GZ, Abelaira HM, Santos MAB, et al. Transtornos depressivos e algumas comorbidades em idosos: um estudo de base populacional. Rev Bras Geriatr Gerontol. 2016:19(1):95-103. doi: 10.1590/1809-9823.2016.14210

25. Laurenti R, Jorge MHPM, Gotlieb SLD. Perfil epidemiológico da morbi-mortalidade masculina. Ciênc Saúde Coletiva. 2005;10(1):35-46. doi: 10.1590/S1413-81232005000100010

26. Berlezi EM, Faria AM, Dallazen F, Oliveira KR, Pillat AP, Fortes CK. Como está a capacidade funcional de idosos residentes em comunidades com taxa de envelhecimento populacional acelerado? Rev Bras Geriatr Gerontol. 2016;19(4):643-52. doi: 10.1590/1809-98232016019.150156

27. Silva NA, Menezes TN. Capacidade funcional e sua associação com idade e sexo em uma população idosa. Rev Bras Cineantropom Desempenho Hum. 2014;16(3):359-70. doi: 10.5007/1980-0037.2014v16n3p359

28. Instituto Brasileiro de Geografia e Estatística. Atlas do Censo Demográfico 2010 [Internet]. Rio de Janeiro: IBGE; 2013 [cited 2019 Oct 3]. Available from: http://censo2010.ibge.gov.br/ apps/atlas/ 